\title{
New genotypes of Trichophyton quinckeanum isolated from cases of human and animal dermatophytosis from Iran
}

Rezaei-Matehkolaei ALI ${ }^{1}$, Ansari, SAHAM ${ }^{2}$, Abastabar, MAHDI ${ }^{3}$

${ }^{1}$ Infectious and Tropical Diseases Research Center, Health Research Institute, Ahvaz Jundishapur

University of Medical Sciences, Ahvaz, Iran; ${ }^{2}$ Shahid Beheshti University of Medical Sciences, Tehran, Iran;

${ }^{3}$ Mazandaran University of Medical Sciences, Sari, Iran.

Background: The formerly Trichophyton mentagrophytes var. quinckeanum is a zoophilic dermatophytes that in the newest taxonomy of dermatophytes has taken species status, referred to as $T$. quinckeanum. We report the first proven cases of human and animal infections with the species from Iran.

Case presentation: the crusts and hairs from a 2-year old female Rüppell's sand fox (Vulpes rueppellii) with some alopecic patches on legs and abdomen (fig. 1a) and also the scales from neck of a 4-year-old boy suspected to dermatophytosis were subjected to direct microscopy with $\mathrm{KOH} 10 \%$ and culture on mycobiotic agar. Direct examination were positive for hyaline septate hyphae and arthroconidia suggestive of dermatophytosis. The colonies had relatively slow growth and showed a white color, domeshaped, cottony in texture and diffused in the margins (fig. 1b). Microscopic examination of culture showed a lot of small, sessile and tear-shaped microconidia and thin wall, cigarette-shape, long and 3-8 cells macroconidia (fig. 1c).

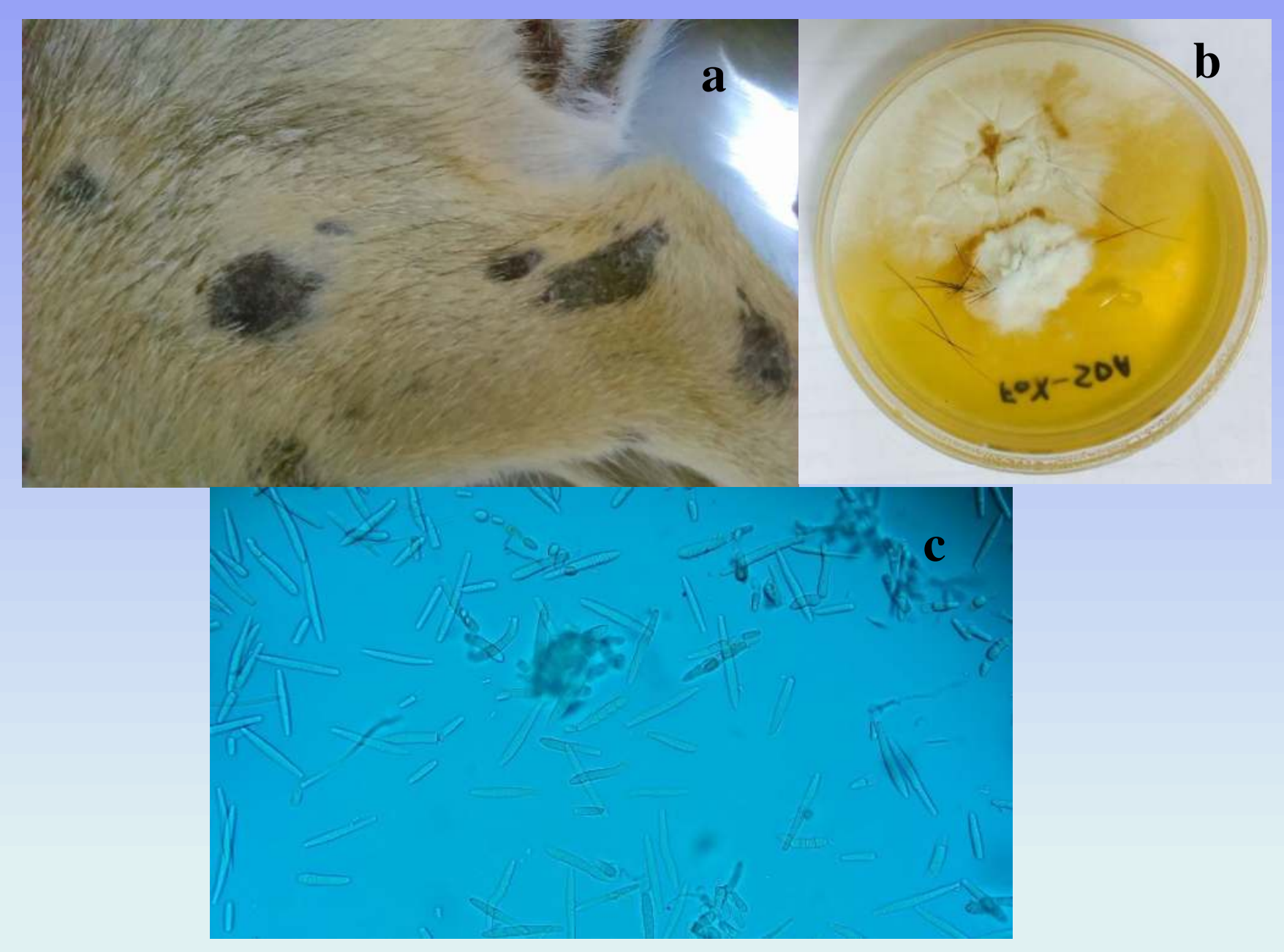

Fig 1. a: alopecic patches on the legs of Rüppell's sand fox, b: (Mcro) and c: (Micro-morphology) of the fox T. quinckeanum isolate, grown on Mycobitic agara after 2 weeks.
Microscopic examination of culture showed a lot of small, sessile and tear-shaped microconidia and thin wall, cigaretteshape, long and 3-8 cells macroconidia (fig. 1c). According to the phenotypic features, the isolate was identified as Trichophyton sp. Amplification of the ITS rDNA, subsequent digestion with Mval enzyme and electrophoresis on $2 \%$ agarose gel led to restriction pattern known for $T$. interdigitale and $T$. simii. However, in PCR-sequencing of the ITS-rDNA both strains showed the most similarity (9999.3\%) to the sequence JQ407219 for T. quinckeanum strain IHEM13697 which recently has designated as the neotype for the species. Surprisingly, in the ITS phylogenetic dendrogram, two isolates were clearly set apart from the reference strains of $T$. quinckeanum (fig. 2). The strains showed potential of new species and should be subjected on more mycological and molecular assessments.

\section{Refference:}

1- Uhrlaß S, Schroedl W, Mehlhorn C, Krüger C, Hubka V, Maier T, Gräser Y, Paasch U, Nenoff P. Molecular epidemiology of Trichophyton quinckeanum-a zoophilic dermatophyte on the rise. JDDG: Journal der Deutschen Dermatologischen Gesellschaft. 2018;16(1):21-32.

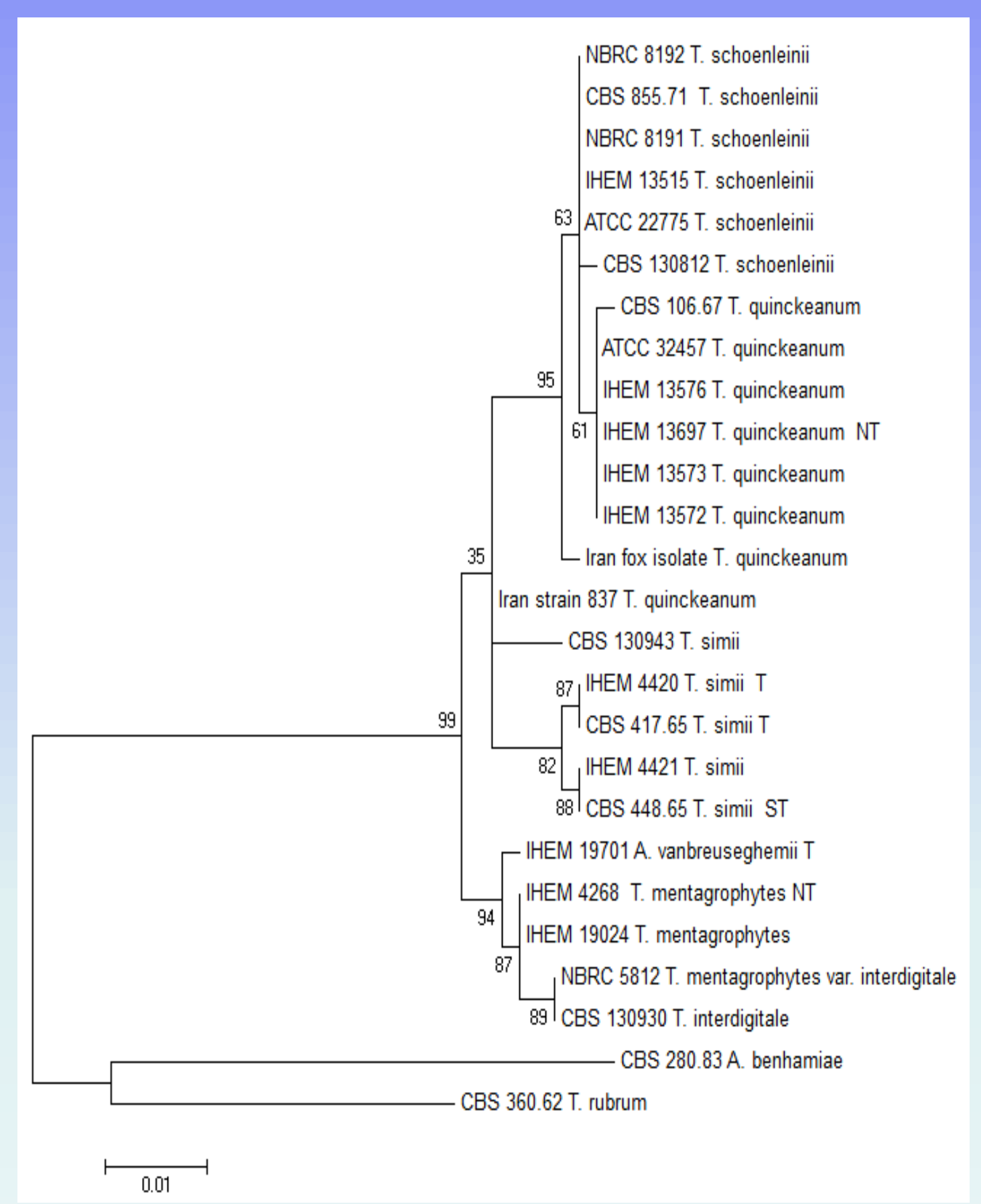

Fig 2. Dendrogram based on sequencing data of the ITS-rDNA region of $T$. quinckeanum, T. schoenleinii, T. simii, T. mentagrophytes and T. interdigitale strains. Statistical method: Maximum likelihood, 1,000 Bootstrap replicates. 